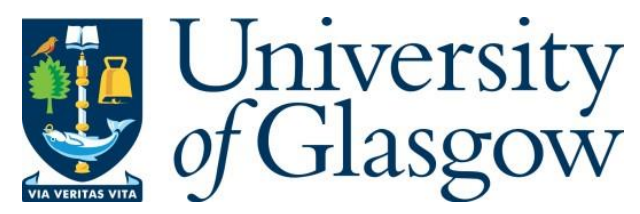

Jaber, M., Owens, D., Imran, M. A., Tafazolli, R., and Tukmanov, A. (2016) A Joint Backhaul and RAN Perspective on the Benefits of Centralised RAN Functions. In: 2016 IEEE International Conference on Communications Workshops (ICC), Kuala Lumpur, Malaysia, 23-27 May 2016, pp. 226-231. ISBN 9781509004485.

There may be differences between this version and the published version. You are advised to consult the publisher's version if you wish to cite from it.

http://eprints.gla.ac.uk/132999/

Deposited on: 1 March 2017

Enlighten - Research publications by members of the University of Glasgow http://eprints.gla.ac.uk 


\title{
A Joint Backhaul and RAN Perspective on the Benefits of Centralised RAN Functions
}

\author{
Mona Jaber ${ }^{1}$, David Owens ${ }^{1,2}$, Muhammad Imran ${ }^{1}$, Rahim Tafazolli ${ }^{1}$, and Anvar Tukmanov ${ }^{3}$ \\ ${ }^{1}$ Centre for Communication Systems Research, University of Surrey, Guildford, GU2 7XH, UK \\ Email: \{m.jaber, m.imran, r.tafazolli, d.owens\}@surrey.ac.uk \\ 2 Telefonica, 260 Bath Road, Slough, Berkshire, SL1 4DX, UK \\ Email: david.owens@telefonica.com \\ ${ }^{3}$ BT Research and Innovation, Adastral Park, Ipswich, IP5 3RE, UK \\ Email: anvar.tukmanov@bt.com
}

\begin{abstract}
Cloud-RAN (C-RAN) is considered a prime enabler to $5 \mathrm{G}$ with promising resource pooling gains, tighter coordination among cells, and cost saving in remote radio heads and corresponding deployment and operation. However, C-RAN brings stringent requirements on the backhaul last mile, or the fronthaul, in terms of capacity, latency, and synchronisation, to the extent that direct fibre is believed to be the only plausible fronthaul solution. Knowing that more often than not, fibre to the home is not available and is a cumbersome and costly technology to provide, what are the alternatives for deploying C-RAN? How much loss is incurred in a 5G network if C-RAN is not available? On the other hand, the distributed RAN (D-RAN) is less demanding on the backhaul but is believed to lack in performance in terms of resource usage and efficiency of RAN deployment. In this work we address the comparison between C-RAN and D-RAN from a joint RAN and backhaul perspective in a quantitative manner, using a case study approach. Our results show that $\mathrm{C}-\mathrm{RAN}$ is indeed cost effective and advantageous from a joint perspective; moreover, intermediate functional splits between the C-RAN and the D-RAN are promising as an evolution path towards $5 \mathrm{G}$, in the absence of fibre.
\end{abstract}

Index Terms-C-RAN, D-RAN, cost analysis, functional split, backhaul, fronthaul

\section{INTRODUCTION}

Cloud or centralised radio access network (C-RAN) is presented as a key disruptive technology, vital to the realisation of $5 \mathrm{G}$ networks. However, C-RAN is currently only feasible with ultra-high bandwidth, very-low latency with highly reliable fronthaul; i.e. optical fibre. The C-RAN is based on splitting the functions of the traditional radio base station and migrating most to a shared pool of processing, called the base band unit (BBU). The radio base station then becomes a simple remote radio head (RRH) and is connected to the BBU through the fronthaul. In this case, how would $5 \mathrm{G}$ evolve in the absence of fibre, knowing that only five countries in Europe have more than $15 \%$ coverage of fibre to the home [1]?

The C-RAN versus D-RAN comparison has been addressed qualitatively in the literature, whereas it requires a quantitative analysis to enable tangible guidelines concerning the "C-RAN versus D-RAN" dilemma. Studies that advocate C-RAN for its superior RAN functionality and significant RAN cost reduction emphasise that it is only feasible with a fibre-based fronthaul; nonetheless, the latter is often unavailable and very expensive and impractical to deploy. On the other hand, there are studies that promote D-RAN because it operates over a realistic backhaul, but warns against the losing the centralisation benefits (cost reduction and ease of deploying RAN features). Various functional splits are also analysed from a fronthaul perspective and resulting reduction in overhead, while highlighting the increase in RRH complexity and the incurred limitation in RAN features. Table I summarises the agreed messages from the C-RAN/DRAN comparison. The gap in these studies is a quantitative comparison of how much is lost and how much is gained with the various RAN architectures, when looking at the problem from a joint backhaul-RAN perspective.

Bhaumik et al. presented the CloudIQ architecture, a first

TABLE I

COMPARISON BETWEEN D-RAN AND C-RAN ARCHITECTURES.

\begin{tabular}{|c|c|c|}
\hline Factor & D-RAN & C-RAN \\
\hline Cost of RRH & High & Low \\
\hline Planning, deployment, maintenance of RRH & High & Low \\
\hline Energy efficiency of RRH & Low $\dagger$ & High \\
\hline Cost of BBU & N/A & High \\
\hline Planning, deployment, maintenance of BBU & N/A & Low $\S$ \\
\hline Energy efficiency of BBU & N/A & High $\dagger$ \\
\hline Potential for resource pooling & Limited & High \\
\hline Fronthaul requirements & Relaxed & Exigent \\
\hline Cost of backhaul/fronthaul $\ddagger$ & High & Higher \\
\hline Level of inter-cell coordination & Limited & Maximum \\
\hline \multicolumn{3}{|c|}{$\begin{array}{l}\text { †On/Off switching in a D-RAN architecture may be used to economise on energy consumption } \\
\text { but due to the complexity of the small cell, each would require additional energy for cooling and } \\
\text { environment control and would still consume more energy when it is ON. The C-RAN RRH has } \\
\text { low complexity, hence is more robust and requires less energy to operate. } \\
\ddagger \text { The cost of the backhaul in a D-RAN architecture is high in view of the additional capillaries } \\
\text { needed to connect all cells to the backbone. For the same scenario the cost of the fronthaul is higher } \\
\text { as a result of higher exigence, thus the need for more bandwidth, less latency, resilience, etc. }\end{array}$} \\
\hline
\end{tabular}

attempt at providing a rigorous resource management framework enabling the trade-off between the quality and cost of deploying/operating the network [2]. Their work, published in 2012, is based on $3 \mathrm{G}$ cellular networks but was able to identify key challenges, namely, the corresponding stringent requirements on the backhaul and the potential solution of functional split. Wang et al. in [3] discuss the potentials of C-RAN in a multi-RAT (radio access technology) network, with the support of network function virtualisation (NFV) and software defined networks (SDN). Besides, they discuss the fronthaul/backhaul challenges and potential solutions, addressing the problem from two perspectives: variable levels of function centralisation and alternative fronthaul technology (e.g., millimetre wave or mmWave). Although the comparison provided is useful, nonetheless, the study remains qualitative and does not provide numerical guidelines. China Telecom have already started deploying C-RAN but are, however, facing challenging fronthaul issues despite the broad availability of optic fibres in the country. In [4], they propose the usage of the next generation fronthaul interface, NGFI, instead of the common public radio interface (CPRI) which is not suitable for $5 \mathrm{G}$. The NGFI is Ethernet-based, hence packet switched and does not naturally support frequency and phase synchronisation; solutions 
are proposed but limitations still exist in the presence of inter-cell coordination techniques such as coordinate multi-point (CoMP). Moreover, jitter and latency remain unsolved difficulties facing the realisation of NGFI. Besides, NGFI also looks at a different functional split in order to alleviate the fronthaul requirements and decouple its dependency on number of antennae, number of users, etc. The NGFI framework may indeed facilitate the realisation of $5 \mathrm{G}$ networks, however, the work presented does not address areas that lack fibre nor does it offer a cost analysis of the solutions proposed. Authors in [5], propose a comparative study of the complexity of various functional splits versus the fronthaul cost, using graph theory and genetic algorithm to find the optimised centralisation option. However, the authors remain at the theoretical level and do not offer tangible comparative results of different fronthaul technologies etc. The RAN as a service (RANaaS) is used in [6] to enable flexible functional split based on fronthaul availability; a study on backhaul requirements and impact on signalling processing is presented for different centralisation levels. In [7], the same authors compare the set of requirements to the various backhaul solutions, putting forward guidelines for fronthaul dimensioning. A theoretic framework is proposed in [8] to evaluate the deployment cost of a network including RAN and backhaul. The model is used to assess the benefits of centralised RAN, considering network costs and RAN gain, with one specific level of centralisation and two possible backhaul technologies: microwave and fibre optics.

In this paper, we present a cost-versus-benefit analysis of different functional splits, considering three types of backhaul technologies: copper-based G.fast ${ }^{1}$, point-to-multi-point microwave, and optical fibre. The study takes on a joint backhaul-RAN perspective and is based on a holistic network dimensioning method using backhaul-aware dynamic cell range extension approach [9]. The paper is structured as follows. Section II introduces the system model. Section III describes the cost and capacity assumptions adopted for different scenarios. Section IV presents the results with analysis and insights. Finally, Section VI summarises the findings and concludes the paper.

\section{SYSTEM MODEL}

In this work, we follow a case-study approach to shed light on the presented open question. The case-study stems from a network engineering perspective with the objective of calculating the minimum number of small cells or RRH to cater for a given traffic load in a defined target area. A backward engineering methodology is followed, whereby, given a fixed number of small cells/RRH, the maximum system throughput is derived. Six possible RAN architectures are considered, ranging from D-RAN to C-RAN, including four functional splits as shown in Figure 1. The centralised BBUs are assumed to be co-located with the macro-cells. Three backhaul technologies are studied: G.fast, microwave, and fibre-based, as shown in Figures 2, 3, and 4, respectively. The dimensioning exercise is holistic covering both radio and backhaul last mile requirements and limitations. The system model assumes that the macro-cell acts as a backhaul aggregator connecting all small cells in the area back to the backbone through an ideal connection (high bandwidth and low latency). The dynamic backhaul-aware cell range extension (CRE) approach in [9] is employed in all cells to self-adjust the

${ }^{1} \mathrm{G}$.fast is a digital subscriber line (DSL) technology; the $G$ stands for the ITU$\mathrm{T} \mathrm{G}$ series of recommendations, whereas fast stands for fast access to subscriber terminals. number of users connected to each small cell based on the radio conditions and backhaul/fronthaul status. CRE is a heterogeneous network feature that aims at biasing users to camp on the small cells by offsetting the cell ranking, traditionally based on the received signal strength at the user terminal. Higher CRE offset values result in a higher number of users served by the small cells and higher area spectral efficiency, given that advanced inter-cell-interference coordination is in place. In this work, the CRE offset is dynamically set, between $0 \mathrm{~dB}$ and $12 \mathrm{~dB}$, using self-optimisation techniques, in a way that backhaul congestion is avoided and total system throughput is maximised. A total of 18 possible deployment scenarios can be realised with the six RAN architectures and three backhaul technologies; many more can be designed if a mix of backhaul technologies is considered. Nine realistic combinations are selected for this study out of the possible 18 to avoid backhauling high overheads over capacity limited links such as G.fast; these deployment scenarios are detailed in Table II.

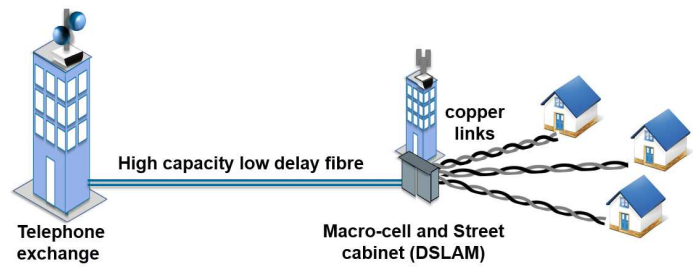

Fig. 2. Last mile of the small cell backhaul employs copper-based G.fast.

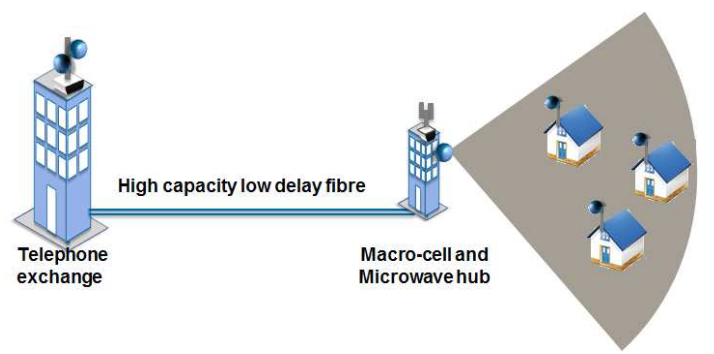

Fig. 3. Last mile of the small cell backhaul is provisioned using is point to multi-point microwave coverage.

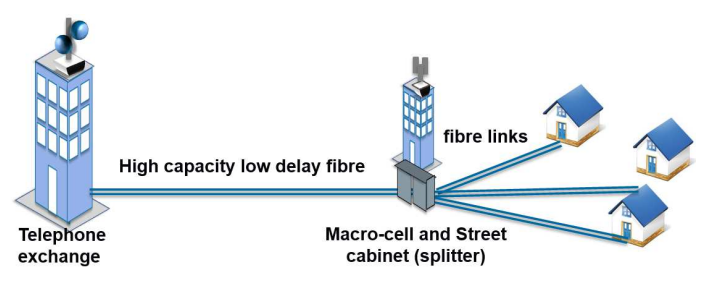

Fig. 4. Last mile of the small cell backhaul is provisioned using fibre to the home technology (FTTH).

\section{CAPACITY AND COST ASSUMPTIONS}

The factors that drive the RAN cost down when functions are centralised are many-fold. Firstly, the complexity and size of the RRH decrease with more centralisation, implying lower equipment cost, alleviated site requirements (e.g., cooling, security, rectifier backup, etc.), and less operational costs, such as maintenance, power bills, and site lease. Also, planning, installation and commissioning costs decrease with less complex 


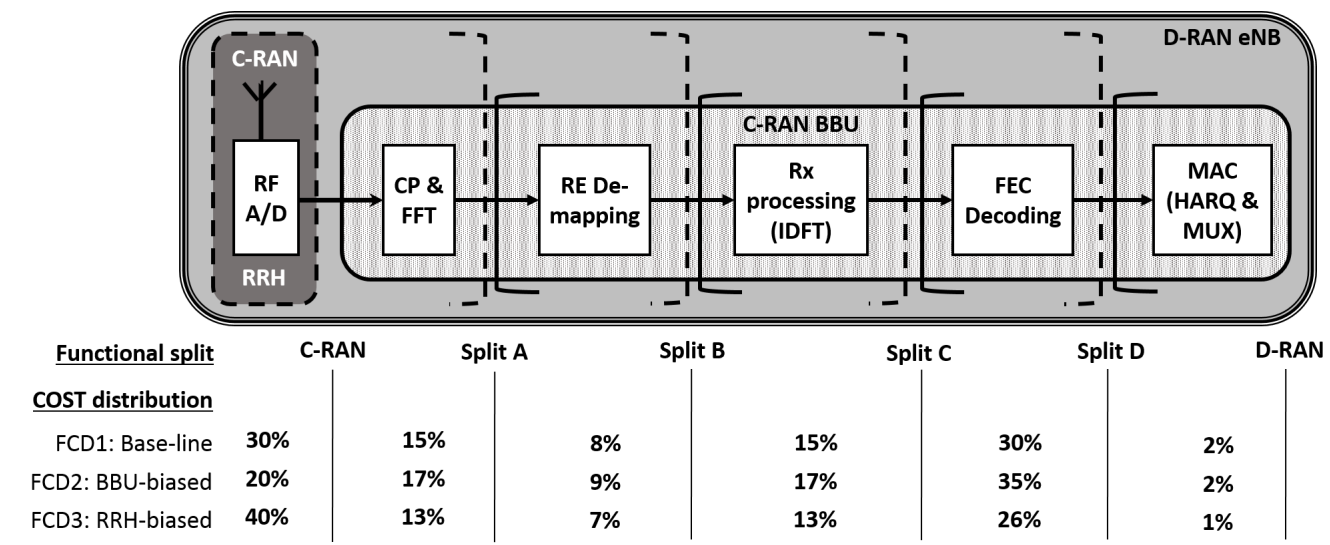

Fig. 1. The C-RAN architecture consists of breaking the eNB into a low complexity RRH and a shared pool of BBU. Various functional splits are also considered in which more functions are kept at the RRH side and less is shared in the BBU. The D-RAN is the traditional RAN architecture in which the eNB includes all of the above functions and connects back to the evolved packet core (EPC) through the backhaul. Three different eNB function cost distributions (FCD) are considered; FCD1 is the baseline assumption based on [2], FCD2 assumes that the cost is biased towards the BBU (advantageous for centralisation), and FCD3 assumes that the cost is shifted towards the RRH (limits centralisation benefits).

TABLE III

COST ASSUMPTIONS RELATED TO RAN AND BACKHAUL CAPITAL (CAPEX) AND OPERATIONAL (OPEX) EXPENDITURES.

\begin{tabular}{|l|l|l|l|}
\hline Item & CapEX $(£)$ & OpEX $(£$ /year) & Remark \\
\hline eNB & 67,000 & 25,000 & {$[11]$} \\
\hline Small cell & $\mathrm{C}_{\mathrm{SC}}=10,000$ & $\mathrm{O}_{\mathrm{SC}=2,450}$ & {$[11]$} \\
\hline RRH & $\mathrm{C}_{\mathrm{SC}} \cdot f(s) \ddagger$ & $\mathrm{O}_{\mathrm{SC}} \cdot f(s)$ & $\begin{array}{l}\mathrm{f}(\mathrm{s}) \text { is the function cost distribution } \ddagger \text { based on }[2] \\
\text { as shown in Figure } 1\end{array}$ \\
\hline Centralised BBU & $\mathrm{C}_{\mathrm{SC}} \cdot(1-f(s)) \S$ & $1 / 3$ of CapEX $\S$ & calculated guess \\
\hline Upgrading central office to support G.fast & 5000 & $10 \%$ of CapEX $\dagger$ & {$[?]$} \\
\hline Cost of lease of 5km copper line & $\mathrm{N} / \mathrm{A}$ & 9600 & order of magnitude in line with [13] \\
\hline Cost of optical network central office & 50,000 & $10 \%$ of CapEX $\dagger$ & calculated guess based on [?] \\
\hline $\begin{array}{l}\text { Cost of fibre/metre (trenching, installing, equip- } \\
\text { ment) }\end{array}$ & 50 & $1 \%$ of CapEX $\dagger$ & calculated guess based on [?] \\
\hline Cost of point of multi point microwave connection & 7,000 & $10 \%$ of CapEX $\dagger$ & calculated guess based on [15] \\
\hline
\end{tabular}

$\S$ The BBUs are dimensioned area-wide and not cell-wide, hence only (12/21) BBUs are needed. The OpEX of the centralised BBU is $1 / 3$ of the same distributed equipment because, the cost of environment control is shared and the cost of maintenance visits is reduced.

$\ddagger f(s)=\sum_{s=1}^{S} w_{s} ; \mathrm{s}$ refers to the function index where $\mathrm{s}=1$ in the RF A/D function and $\mathrm{s}=6$ is the MAC function. $w_{s}$ refers to the function corresponding weight e.g., under FCD1, $w_{1}=30 \%$ and $w_{6}=2 \%$ (see Figure 1). $S$ refers to the dynamic functional split, e.g., for Split B $S=3$, indicating that the RRH in this architecture includes the first three eNB functions.

$\dagger$ These values are calculated assumptions, but are not supported by any reference.

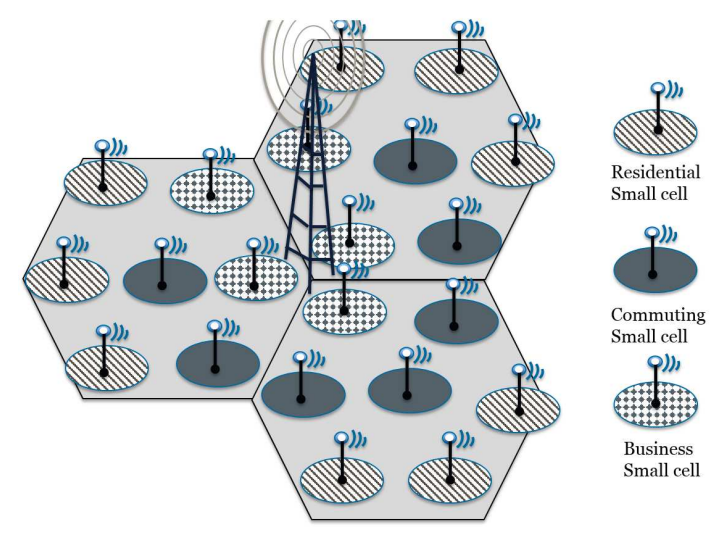

Fig. 5. The system is a heterogeneous network including macro-cells and small cells. There are three types of small cells with different peak traffic time.

TABLE II

SCENARIOS CONSIDERED IN THE STUDY (REFER TO FIGURE 1).

\begin{tabular}{|l|l|l|}
\hline Scenario & Functional split & Backhaul technology \\
\hline 1 & D-RAN- Traditional eNB & G.fast \\
\hline 2 & Split D- MAC forwarding & G.fast \\
\hline 3 & Split D- MAC forwarding & Microwave \\
\hline 4 & Split C- Soft bit forwarding & G.fast \\
\hline 5 & Split C- Soft bit forwarding & Microwave \\
\hline 6 & Split B- Rx Data forwarding & Microwave \\
\hline 7 & Split A- Sub-frame forwarding & Microwave \\
\hline 8 & Split A- Sub-frame forwarding & Fibre-based \\
\hline 9 & C-RAN- CPRI - I/Q forwarding & Fibre-based \\
\hline
\end{tabular}

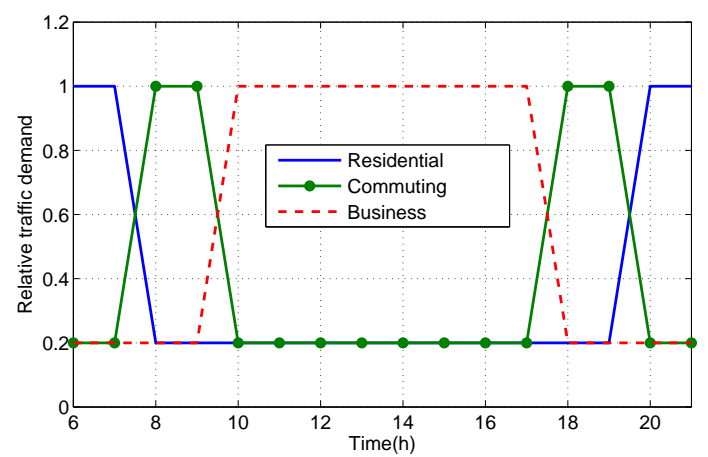

Fig. 6. There are three types of cells: residential, transportation, and business district. Each has a different peak traffic pattern depending on the time of the day. It is assumed that there is no overlap between different cell types' peak time; also, the off-peak traffic is $20 \%$ of the peak traffic.

RRHs, because they become easier to plug-and-play, and are often part of an area-wide deal (e.g. lamp posts, gas stations, billboards, etc.), hence, do not require individual planning and site acquisition efforts. Moreover, centralisation improves resource pooling, especially in a situation where the peak traffic of small cells occurs at different times. For instance, if the peak traffic of the total area is known, the base band capacity would be dimensioned according to it, irrelevant of how the bulk of the traffic moves within the area. Whereas in a D-RAN deployment, each small cell should be provisioned independently to cater for its forecast peak traffic, knowing that at other times of the day 
the resources will be under-utilised. Even with advanced features, such as putting small cells to sleep mode when the traffic is low, the base band resources should still be installed in the small cells, thus driving the capital expenditure (CapEX) up. The difficulty in this analysis stems from tagging a realistic relative cost to each of these separate factors. The base-line assumptions considered refer to published material where possible, and are summarised in Table III. The total cost of ownership (TCO) of each scenario is computed by adding the CapEX to the operational expenditure (OpEX) over 5 years. The capacity of the various backhaul technologies is detailed in Table IV. The D-RAN small cell, as priced in Table III, is assumed to have enough base band capacity to cater for the peak traffic in a hot-spot. Moreover, the relative backhaul overheads per functional split considered are computed in a similar approach as in [10].

TABLE IV

CAPACITY ASSUMPTIONS OF BACKHAUL TECHNOLOGIES CONSIDERED IN THE STUDY.

\begin{tabular}{|c|c|c|}
\hline Item & Capacity & Remark \\
\hline G.fast & relative to distance & {$[16]$} \\
\hline $25 \mathrm{~m}$ & 600Mbps & \\
\hline $50 \mathrm{~m}$ & 420Mbps & \\
\hline $75 \mathrm{~m}$ & 390Mbps & \\
\hline $100 \mathrm{~m}$ & 260Mbps & \\
\hline $125 \mathrm{~m}$ & 240Mbps & \\
\hline $150 \mathrm{~m}$ & 220Mbps & \\
\hline $175 \mathrm{~m}$ & 210Mbps & \\
\hline $200 \mathrm{~m}$ & 200Mbps & \\
\hline $225 \mathrm{~m}$ & 180Mbps & \\
\hline $250 \mathrm{~m}$ & $175 \mathrm{Mbps}$ & $>250 \mathrm{~m} \mathrm{100Mbps}$ \\
\hline Microwave & 600Mbps & {$[17]$} \\
\hline Fibre-based & 10Gbps & assuming ideal backhau \\
\hline
\end{tabular}

\section{RESULTS AND ANALYSIS}

Each of the scenarios in Table II is implemented in a Matlabbased system simulator, over the period depicted in Figure 6, to obtain the corresponding reachable effective system throughput. If the backhaul/fronthaul link is limiting in a cell, the effective throughput would correspond to the maximum possible throughput over that link. On the other hand, if the radio interface is the throughput bottleneck, the effective throughput would be the cumulative radio throughput. In each scenario, the number of users is increased until saturation; the corresponding effective system throughput designates the design's capacity.

The total effective throughput, as perceived by users in the network, is shown in Figure 7 for each of the deployment scenarios. There are two factors impacting the effective throughput: the functional split and the backhaul technology. The functional split results in different backhaul overhead, hence may limit (or ease) the effective throughput, due to backhaul constraints. This is evident when we compare Scenario 3 to Scenarios 5, 6, and 7, all employing microwave links for backhaul with increasing overhead due to the functional split. Scenarios 5, 6, and 7 are crippled with the backhaul overhead, resulting in $\sim 25 \%$ less effective throughput in Scenario 7 compared to Scenario 3. The backhaul technology is another factor limiting the effective throughput, due to its capacity constraints, such as Scenarios 7 and 8 which have the same RAN architecture with microwave backhaul and fibre, respectively. Scenario 7 is severely limited by the backhaul capacity whereas Scenario 8 allows $36 \%$ more effective throughput.

Another point worth mentioning is that, in the example selected, the fronthaul capacity does not increase linearly, but

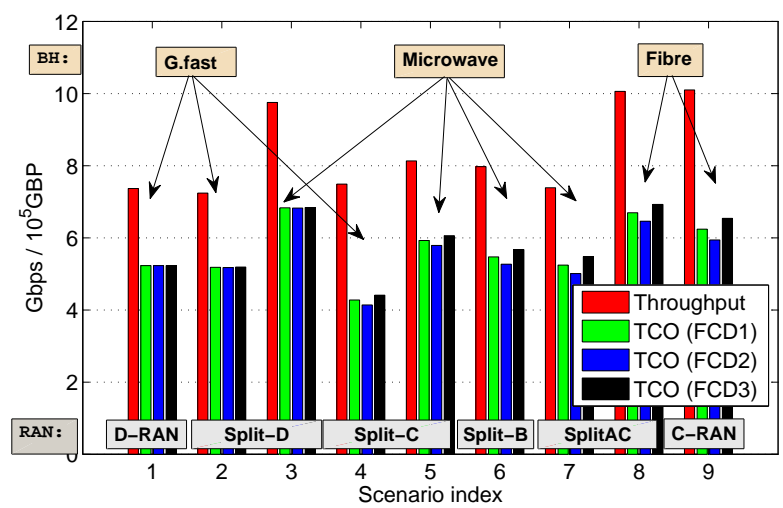

Fig. 7. The cumulative effective throughput, as perceived by users, is computed in each deployment scenario and shown in red bars, expressed in Gbps. This is compared to the corresponding TCO under three different function cost distributions (FCD), shown in Figure 1, expressed in 100,000GBP. The x-axes indicates the scenario index, as in Table II, and the corresponding RAN functional split and backhaul solution choice are highlighted for clarity.

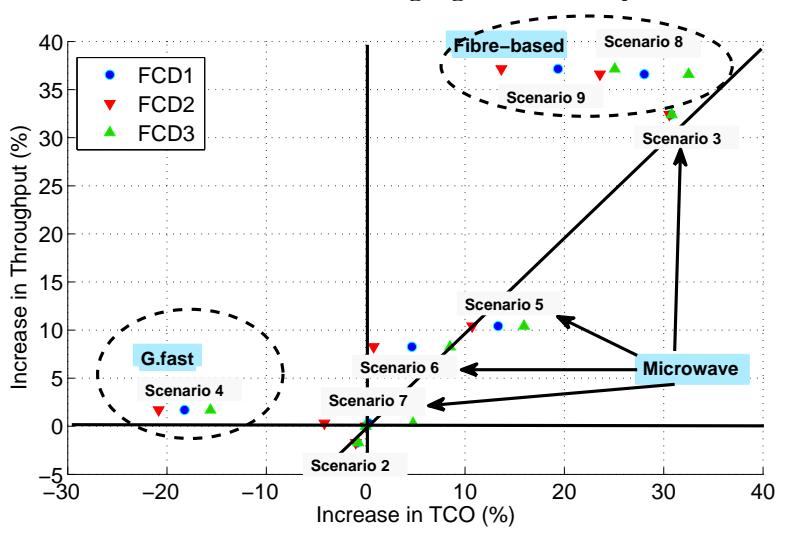

Fig. 8. The increase/decrease in effective throughput of each scenario, relative to Scenario 1 , is compared to the corresponding increase/decrease in TCO for the three different FCD assumptions. The diagonal line separates the profitable and non-profitable regions; scenarios that fall below the line indicate higher increase in TCO than in throughput. Scenarios 2, 5, and 7 are not profitable since they lay on or below the diagonal line.

jumps from limited capacity of G.fast connections, to 600Mbps of microwave, to $10 \mathrm{Gbps}$ of fibre. For this reason, the fronthaul is often over-dimensioning such as in scenarios 3 and 8 . In these scenarios, reducing the fronthaul capacity by one step results in severe performance degradation as seen in Scenarios 2 and 7. Accordingly, intermediate backhaul solutions that fill the gaps between G.fast and microwave, and microwave and fibre, are needed. High bit rate in-band fronthaul could be such a solution or wireless mmWave fronthaul, with variable capacity as detailed in [18], a commercial mmWave solution for the fronthaul.

Figure 8 displays the gains/losses of each of the eight deployments scenarios featuring variable levels of centralisation, compared to the D-RAN. Scenario 1, D-RAN with G.fast, acts as a benchmark; the capacity gains/losses of all other scenarios are derived by comparing their respective cumulative effective throughput to the baseline. In parallel, the increase/decrease in TCO of each scenario is defined with respect to Scenario1. The diagonal line separates the region of advantageous from the unprofitable scenarios; those that fall on the line incur comparable cost increase and capacity gain, those below the line are dominated by cost, whereas those above have higher capacity gains. It can be observed from Figures 7 and 8 that the gain of centralisation in Scenarios 8 and 9 is more important than the extra cost of fibre. Thus, these results answer our open 
question partly: a joint perspective of C-RAN with fibre-based fronthaul reveals that the cost reduction of function centralisation overtakes the cost of fibre, hence is cost effective as opposed to the common belief that the C-RAN gain was met with debilitating fibre costs. Moreover, profitable scenarios are identified, where a scenario is considered profitable if the effective throughput gain is higher than the TCO increase, relative to the benchmark Scenario 1. Scenarios 2, 5, and 7 are not profitable since the incurred additional cost compares with the increase in throughput, hence are not justified. Scenario 4 is the most cost-efficient among the G.fast-based solutions because it offers a reduction in cost due to centralisation, while compensating for reduction is small cell capacity by shifting users to the available macro layer, thus resulting in unaltered cumulative throughput. Moreover, functional splits Split B, Split A and C-RAN may not be supported with G.fast; whereas, with microwave, the only options are D-RAN and Split D due to the high cost of microwave that cancels out the centralisation gain.

Although these results cannot be conclusive, since they depend on delicate cost assumptions; nonetheless, some useful insights can be drawn. In the presented case-study, the highest gain reached with centralisation is $37 \%$ increase in effective throughput; on the other hand, the highest increase in cost is $27 \%$, under FCD1 assumptions. Thus, the gain from centralisation dominates the increase in TCO, even when fibre to the cell is assumed. But perhaps a more critical factor than cost is the practicality of laying fibre, which is difficult to capture in the analysis. Moreover, the cost distribution of the various functions in the eNB does not alter the results considerably, and is minimal for Scenarios 1 (D-RAN), 2 (Split D), and 3 (Split-C).

\section{SENSITIVITY ANALYSIS}

There are many factors affecting the suitability of each of the considered deployment scenarios; to this end, in this section, we conduct a sensitivity analysis by varying key factors and measuring their impact on the profitability of each scenario. Some of the cost assumptions related to the backhaul technologies vary widely, such as the cost of fibre in different countries, or the cost of microwave deployment in different environments and various commercial solutions. The effect of fibre and microwave cost variations is studied in Section V-A and V-B, respectively. Traffic diversity is another critical aspect that may amplify or reduce the centralisation gains; this is also captured in Section V-C.

\section{A. Impact of microwave cost variation}

In this section, the CapEX and OpEX of microwave backhaul solution is multiplied by a coefficient varying between 0.5 and 1.5, as shown in Figure 9. The figure compares the increase in TCO of Scenarios 3, 5, 6, and 7, employing microwave links, to the corresponding increase in effective throughput relative to the benchmark. The only variable in Figure 9 is the cost of the backhaul, hence, the throughput gain is constant and is shown in horizontal lines. Values of the cost coefficient that increase the TCO towards the right of the intersection points result in unprofitable deployment solution since they cause an increase in cost higher than the throughput gain. It is clear that the microwave-based deployment scenarios are borderline with the assumed coefficient 1 , whereas a $10 \%$ decrease in backhaul cost would render all these scenarios beneficial. Since the microwave solution adopted in this case study is a point to multi-point

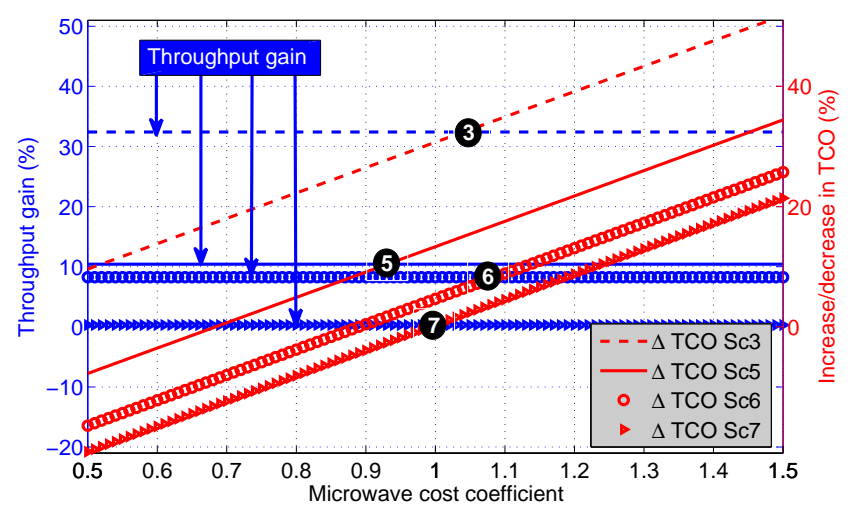

Fig. 9. The increase in TCO (red lines) of the microwave-based deployment scenarios is compared to the corresponding increase in effective throughput (blue lines) relative to Scenario 1, for variable microwave cost coefficient. The intersection points of the TCO and throughput lines correspond to the cost coefficient that results in equal increase in cost and throughput; higher values render the scenarios unprofitable. The baseline FCD1 is considered.

design, the cost per link will scale down with the increase in number of small cells; thus a $10 \%$ reduction in cost is feasible without necessitating changes in commercial agreements.

\section{B. Impact of optical fibre cost variation}

In this section, the capital and operational expenditures of optical fibre backhaul solution is multiplied by a coefficient varying between 0.5 and 1.5 as shown in Figure 10. The figure compares the increase in $\mathrm{TCO}$ of Scenarios 8 and 9, relative to the benchmark, to the corresponding increase in effective throughput. The only variable in Figure 10 is the cost of the backhaul, hence, the throughput gain is constant and is shown in horizontal lines. The fibre-based Scenarios 8 and 9 remain profitable when the cost of fibre is increased by a coefficient of 1.14 and 1.25 , respectively. This is highlighted by the numbered dots at the intersections points, indicating the coefficient value that results in equal increase in throughput and TCO. Deployment cost of backhaul differs considerably among countries; an Analysys-Mason study in the U.K. found that deploying FTTH across the country would cost five times more than deploying FTTC with VDSL; in our assumptions the TCO of deploying fibre in the network is 6.5 times the cost of G.fast [19]. The fibre backhaul cost that matches the results in [19] corresponds to a coefficient $\alpha=0.76$. The increase in TCO for Scenario 9 at this value is minimal compared to the benchmark Scenario 1; a further reduction of $\alpha=0.6$ is needed to bring the cost of Scenario 8 to the same level as Scenario 1.

\section{Impact of traffic diversity}

Another critical factor that affects the suitability of the deployment scenarios is the traffic diversity. The traffic assumptions shown in Figure 6 consider that different small cell types will have distinct peak hour traffic periods. If, on the other hand, less diversity were assumed, i.e., higher probability of different cell types peaking simultaneously, it would necessitate larger BBU resources at the central office and potentially higher operational costs. This situation is captured in Figure 11, Less diversity, and is shown to drive the TCO of all centralised scenarios higher. The opposite case is also considered, which assumes more diversity, i.e. less simultaneous capacity demand in different cells, hence less BBU resources and OpEX. It can be seen that, although the traffic diversity affects the TCO of all centralised scenarios, 


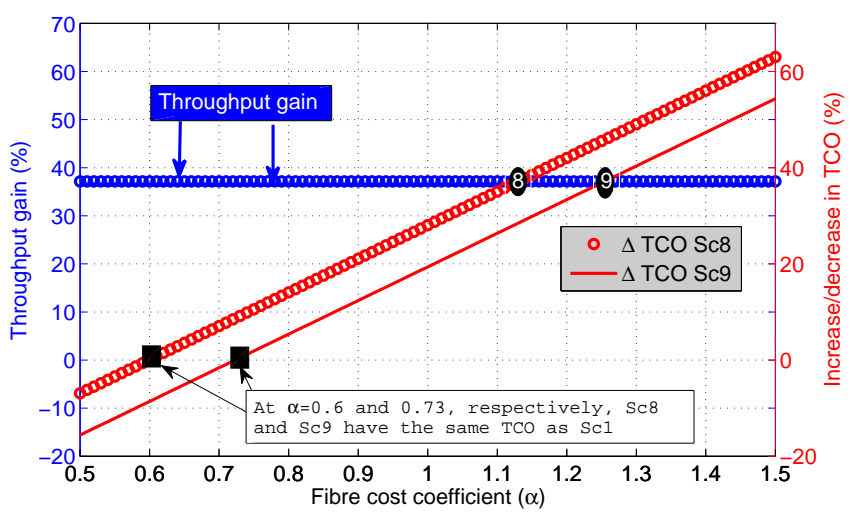

Fig. 10. The increase in TCO (red lines) of the fibre-based deployment scenarios is compared to the corresponding increase in effective throughput (blue lines) relative to Scenario 1, for variable fibre cost coefficient $(\alpha)$. C-RAN with fibre (Scenario 9) remains cost-effective with a cost increase of $25 \%$ and is comparable to D-RAN with G.fast (Scenario 1) for $\alpha=0.76$, the cost assumption in [19]. The baseline FCD1 is considered.

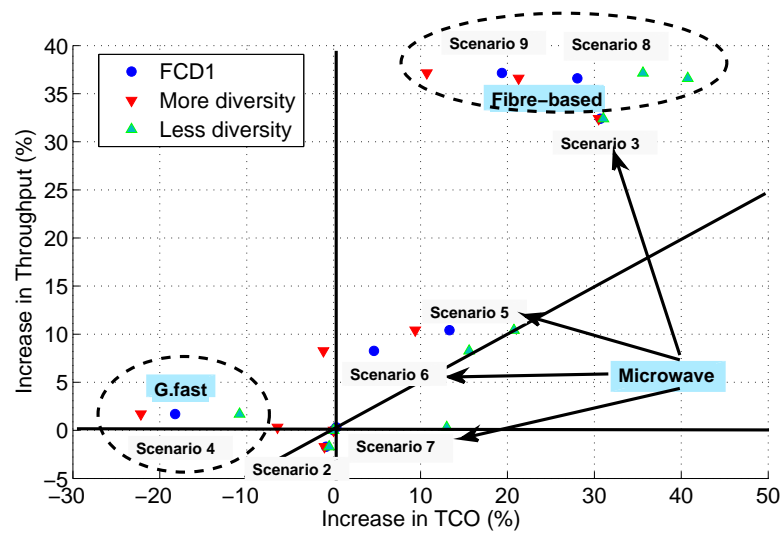

Fig. 11. In this sensitivity analysis, the baseline function cost distribution, FCD1, is considered and different traffic diversity levels are accounted for in the BBU dimensioning and resulting TCO. The same categorisation deduced in Figure 8 for FCD1 remains valid.

it does not however alter their corresponding grading between profitable and unprofitable. In other words, scenarios identified as beneficial in Figure 8 remain so with traffic variation, and vice-versa, though they become less or more costly.

\section{CONCLUSION}

We have presented a case-study based analysis of the gains of centralisation from a joint backhaul-RAN perspective with various functional splits and backhaul solutions. The main message that can be drawn from this study is that RAN centralisation is advantageous for reducing the RAN cost, on one hand, and that fibre, while incurring the highest TCO among backhaul technologies, allows nonetheless a significant increase in throughput, rendering the C-RAN with fibre fronthaul (Scenario 9) the most cost effective solution from a joint backhaul-RAN perspective. Moreover, the analysis proved that Scenario 9 remains a winner when subjected to extreme conditions such as BBU-biased cost distribution of eNB functions (FCD3), increase in cost of optical fibre (up to $1.25 \mathrm{X}$ ), and decrease in traffic diversity $(50 \%$ increase in BBU CapEX and OpEX).

On the other hand, the results advocate the need for a heterogeneous backhaul/fronthaul with variable performance and cost to cater for different small cell needs. Besides, a fronthaul solution that is shared among small cells, such as point-to-multipoint microwave, becomes advantageous in deployments with high numbers of RRH with diverse peak hour traffic distribution. With the three backhaul solutions selected for this case-study, it is not possible to tailor the fronthaul provisioning according to the RRH requirements, leading to excess fronthaul capacity in many scenarios, at an extra cost. Consequently, it would also be interesting to explore other fronthaul solutions with dynamic and flexible bandwidth allocation, e.g., in-band wireless or mmWave, to avoid over-dimensioning the last mile links.

\section{ACKNOWLEDGMENT}

The views expressed here are those of the authors and do not necessarily reflect those of the affiliated organisations. The authors would like to thank the UK Engineering and Physical Science Research Council (EPSRC) and BT Research and Innovation for funding this research through an Industrial Cooperative Awards in Science \& Technology (iCASE) studentship.

\section{REFERENCES}

[1] F. Barros, K. Ahl, and V. Chaillou, "Creating a connected continent: Press conference Warsaw." [Online], Available: http://www.fthcouncil.eu/, 2015.

[2] S. Bhaumik, S. P. Chandrabose, M. K. Jataprolu, G. Kumar, A. Muralidhar, P. Polakos, V. Srinivasan, and T. Woo, "CloudIQ: a framework for processing base stations in a data center," Aug. 2012.

[3] R. Wang, H. Hu, and X. Yang, "Potentials and challenges of C-RAN supporting Multi-RATs toward 5G mobile networks," IEEE Access, vol. 2 , pp. 1187-1195, 2014.

[4] C.-L. I, Y. Yuan, J. Huang, S. Ma, C. Cui, and R. Duan, "Rethink fronthaul for soft ran," IEEE Communications Magazine, vol. 53, no. 9, pp. 82-88, 2015 .

[5] J. Liu, S. Zhou, J. Gong, Z. Niu, and S. Xu, "Graph-based framework for flexible baseband function splitting and placement in C-RAN," arXiv preprint arXiv:1501.04703, 2015.

[6] D. Wubben, P. Rost, J. Bartelt, M. Lalam, V. Savin, M. Gorgoglione, A. Dekorsy, and G. Fettweis, "Benefits and impact of cloud computing on 5G signal processing," IEEE Signal Processing Magazine, vol. 31, pp. 35 - 44, Nov. 2014.

[7] J. Bartelt, P. Rost, D. Wubben, J. Lessmann, B. Melis, and G. Fettweis, "Fronthaul and backhaul requirements of flexibly centralized radio access networks," IEEE Wireless Communications, vol. 22, pp. 105-111, Oct. 2015.

[8] V. Suryaprakash, P. Rost, and G. Fettweis, "Are heterogeneous cloud-based radio access networks cost effective?," IEEE Journal on Selected Areas in Communications, vol. 33, pp. 2239-2251, Oct 2015.

[9] M. Jaber, M. Imran, R. Tafazolli, and A. Tukmanov, "An adaptive backhaulaware cell range extension approachan adaptive backhaul-aware cell rafnge extension approach," in IEEE International Conference on Communications (ICC), Workshops, (London, UK), Jun. 2015.

[10] A. Maeder, M. Lalam, A. de Domenico, E. Pateromichelakis, D. Wubben, J. Bartelt, R. Fritzsche, and P. Rost, "Towards a flexible functional split for cloud-RAN networks," in European Conference on Networks and Communications (EuCNC), (Bologna), Jun. 2014.

[11] M. Paolini, "The economics of small cells and Wi-Fi offload." [Online], Available: http://www.senzafiliconsulting.com/, 2012.

[12] M. Jensen, R. Nielsen, and O. Madsen, "Comparison of cost for different coverage scenarios between copper and fiber access networks," in The 8th International Conference Advanced Communication Technology, ICACT, vol. 3, pp. 2015-2018, Feb 2006.

[13] J. Allen and F. Chevalier, "Report for Vodafone - mobile backhaul market: Phase 2 report," May, 2014.

[14] T. Naveh, "Mobile backhaul: fiber vs. microwave: Case study analyzing various backhaul technology strategies," white paper, Ceragon Network Ltd, 2009.

[15] M. Paolini, "Crucial economics for mobile data backhaul: An analysis of the total cost of ownership of point-to-point, point-to-multipoint, and fibre options," 2012.

[16] M. Timmers, M. Guenach, C. Nuzman, and J. Maes, "G.fast: evolving the copper access network," IEEE Communications Magazine, vol. 51, pp. 7479, Aug. 2013.

[17] Cambridge Broadband Networks, "Vectastar platform introduction." [Online], Available: http://cbnl.com/vectastar-platform-introduction.

[18] MIMOtech (Pty) Ltd, "Janus 70/80G AirDuplex ultra high capacity mmWave fibre extension." [Online], Available: http://www.mimotechnology.com/, 2015.

[19] Analysys Mason, "Final report for the Broadband Stakeholder Group: The costs of deploying fibre-based next-generation broadband infrastructure." [Online], Available: http://www.analysysmason.com/, Sep. 2008. 